Research in Astronomy and Astrophysics manuscript no.

(LTEX: 2020-0354.tex; printed on February 10, 2021; 9:20)

\title{
Simulation of the orbit and spin period evolution of the double pulsars PSR J0737-3039 from their birth to coalescence induced by the gravitational wave radiation
}

\author{
Peng Liu ${ }^{1}$, Yi-Yan Yang ${ }^{2}$, Jian-Wei Zhang ${ }^{3}$ and Maria Rah ${ }^{4}$ \\ ${ }^{1}$ School of Physics and Electronic Engineering, Qilu Normal University, Jinan 250200, China; \\ liupeng_mail@sina.cn \\ 2 School of Physics and Electronic Sciences, Guizhou Education University, Guiyang 550018, China \\ 3 Astronomy Department, Beijing Normal University, Beijing 100875, China \\ ${ }^{4}$ Research Institute for Astronomy and Astrophysics of Maragha(RIAAM)-Maragha, IRAN \\ Received 20xx month day; accepted 20xx month day
}

\begin{abstract}
The complete orbital and sin period evolutions of the double neutron star (NS) system PSR J0737-3039 are simulated from the birth to coalescence, which include the two observed radio pulsars classified as primary NS PSR J0737-3039A and companion NS PSR J0737-3039B. By employing the characteristic age of PSR J0737-3039B to constrain the true age of the double pulsar system, the initial orbital period and initial binary separation are obtained as $2.89 \mathrm{hrs}$ and $1.44 \times 10^{6} \mathrm{~km}$, respectively, and the coalescence age or the lifetime from the birth to merger of PSR J0737-3039 is obtained to be $1.38 \times 10^{8} \mathrm{yr}$. At the last minute of coalescence, corresponding to that the gravitational wave frequency changes from $20 \mathrm{~Hz}$ to $1180 \mathrm{~Hz}$, we present the binary separation of PSR J0737-3039 to be from $442 \mathrm{~km}$ to $30 \mathrm{~km}$, while the spin periods of PSR J0737-3039A and PSR J0737-3039B are $27.10 \mathrm{~ms}$ and $4.63 \mathrm{~s}$, respectively. From the standard radio pulsar emission model, before the system merged, the primary NS could still be observed by the radio telescope, while the companion NS has crossed the death line in the pulsar magnetic-field versus period $(B-P)$ diagram which is usually considered to cease the life as a pulsar. It is for the first time that the whole life evolutionary simulation of the orbit and spin periods for double NS system is presented, which provides the useful information for observing the primary NS at the coalescence stage.
\end{abstract}

Key words: PSR J0737-3039: double neutron star: pulsar: gravitational wave: simulation:

In 1974, Taylor and Hulse discovered the first double neutron star (DNS) system PSR J1913+16, using the Arecibo telescope (Hulse \& Taylor 1975), by which, the existence of gravitational waves (GW) 
\& Sitzungsber 1916; Taylor \& Weisberg 1982). More than 40 years later, for the first time, LIGO and Virgo detected GW directly in the merge of DNS system of an old elliptical galaxy, named as GW170817. Together with a serials of discoveries of the coalescence between the stellar black holes and NSs, the GW theory has been confirmed and the multi-messenger astronomy is out of birth (Abbott et al. 2017; Troja et al. 2017; Blanchard et al. 2017). Therefore, the investigation of the whole evolutionary process of the DNS system from its birth to coalescence and clarifying the details of its evolution is a matter of concern to some scientists working on NS and pulsar astrophysics.

Among the 19 discovered DNS systems until now, PSR J0737-3039 is the only known DNS system in which primary and companion NSs have been detected as pulsars (Burgay et al. 2003; Lyne et al. 2004), which can not only provide the best direct test for the correctness of general relativity, but also can be employed as a natural lab for studying the plasma physics and strong field gravitation (Kramer et al. 2004, 2006; Kramer \& Wex 2009). Only PSR J0737-3039 in 19 DNSs can provide more information to let us know the initial orbital and initial NS spin periods, therefore the PSR J0737-3039 system can be studied as a complete evolutionary sample of the DNS systems from the birth to the merger. PSR J07373039 has a very compact orbit with the orbital period of only $2.45 \mathrm{hrs}$, and the eccentricity is as small as 0.088 (approximately a circular orbit) (Lyne et al. 2004). From the DNS formation model (Bhattacharya \& van den Heuvel 1991; van den Heuvel 2004), the primary NS (PSR J0737-3039A) was the first NS to be formed, generated by a massive star directly through a supernova explosion and experienced accretion induced spun-up and magnetic field decay, with the spin period of $22.7 \mathrm{~ms}$ and the dipole magnetic field of $6.4 \times 10^{9} \mathrm{G}$ (Lyne et al. 2004); the companion NS (PSR J0737-3039B) was the second NS produced by a electron capture supernova explosion (Podsiadlowski et al. 2004; Nomoto 1984) and ultra-stripped supernova explosion two methods (Tauris et al. 2013, 2015, 2017), with the spin period and the dipole magnetic field of $2773.46 \mathrm{~ms}$ and $6.4 \times 10^{9} \mathrm{G}$, respectively (Lyne et al. 2004).

As expected, the orbits of the DNSs are contracted continuously by GW radiation and eventually go to merge, the direct coalescence observation and calculations of which was pointed out by Schutz (Schutz 1986), and by Cutler (Cutler et al. 1992). Now, the numerical relativity is a very powerful tool for studying DNS orbital evolution, when the DNS system enter the merger (Maione et al. 2016; Shibata \& Uryu 2000). In order to study the complete evolution of PSR J0737-3039, we deduced the variation of the orbital period given by Peters (Peters 1964) to obtain a new evolution formula and applied it to simulate the orbital evolution of DNS. Since PSR J0737-3039B is a non-recycled pulsar like Crab pulsar (Lyne \& GrahamSmith 2012; Yang et al. 2006), and the current spin period is much larger than its initial spin period, therefore we can use its characteristic age to approximate the true age of PSR J0737-3039 system (Camilo et al. 1994; Lorimer et al. 2007). Next, we derived the initial orbital period of the system and the initial spin periods of the two pulsars, based on which the simulating of complete evolution of the system is presented.

The paper is organized as bellow: In section 2, we introduce both of the orbital evolution formula of DNS caused by gravitational radiation, then the simulation of the orbital complete evolutions of PSR J07373039 system was given in section 3. We simulate the entire spin period evolution of PSR J0737-3039A and PSR J0737-3039B in section 4. Finally, we simulate the gravitational wave frequency of the last minute 


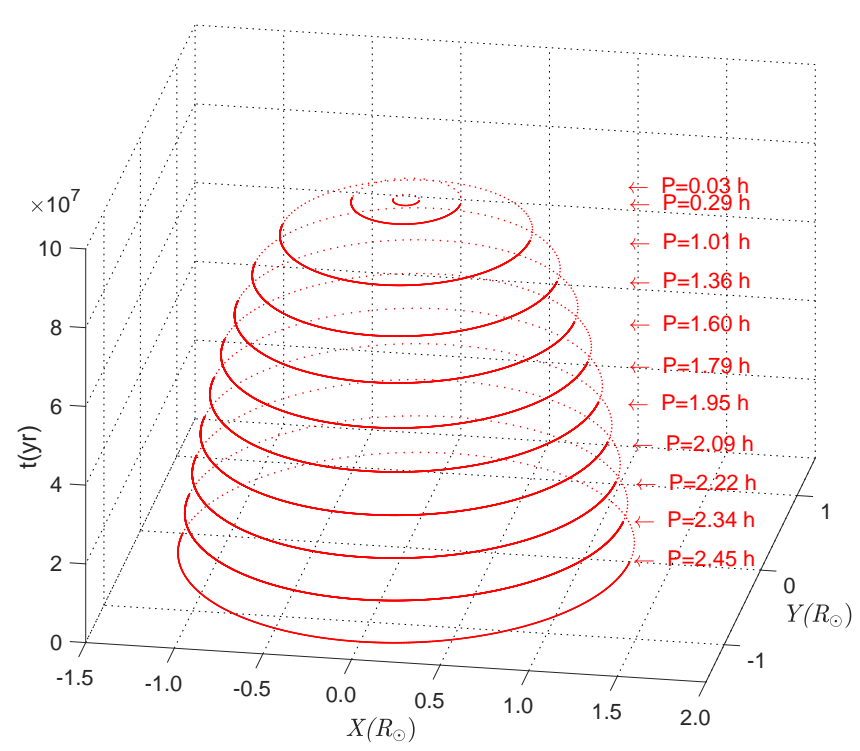

Fig. 1: 3-D diagram of PSR J0737-3039 evolutionary trajectory by GW radiation, where the vertical axis stands for the time in the units of $10^{7} \mathrm{yrs}$ and two horizontal axes of the orbital plane stands for the DNS orbit, with the units of radius of Sun $R_{\odot}=7 \times 10^{10} \mathrm{~cm}$.

\section{ORBITAL EVOLUTION OF DOUBLE NEUTRON STARS}

In this section, we derive the contraction evolution of DNS orbit by GW radiation, based on the variation of the orbit radius (a) of DNS system, which is given below (Peters 1964; Lyne \& Graham-Smith 2012; Shapiro \& Teukolsky 1983; Lightman et al. 1975; Lightman \& Shapiro 1975; Ohanian \& Ruffini 1994):

$$
\frac{1}{a} \frac{d a}{d t}=-\frac{64}{5} \frac{G^{3} M^{2} \mu}{c^{5} a^{4}} f(e)
$$

where $M$ is the total mass of DNS, expressed as $M=M_{p}+M_{c}$ with $M_{p}$ and $M_{c}$ the mass of the primary and companion NS, respectively; $G(c)$ is the gravitational constant (speed of light); $\mu$ is the reduced mass of DNS, expressed as: $\mu=M_{p} M_{c} /\left(M_{p}+M_{c}\right) ; f(e)$ is a function of the orbital eccentricity $e$ of DNS, described in the following (Peters \& Mathews 1963):

In this paper, we only consider the case of circular orbit $e=0$ because the eccentricity of PSR J07373039 is 0.088 , which arises the eccentricity function to be $f(e=0)=1$.

$$
f(e)=\left(1+\frac{73}{24} e^{2}+\frac{37}{96} e^{4}\right)\left(1-e^{2}\right)^{-\frac{7}{2}}
$$

According to the Keplerian motion, the relationship between the DNS orbital period and the binary separation is:

$$
P=\left(\frac{a^{3} 4 \pi^{2}}{G M}\right)^{\frac{1}{2}}
$$

thus the orbital evolution can be also described by the orbital period equivalently. We bring Eq.(2) and Eq.(3) into Eq.(1) and solve the evolution of DNS binary separation with time, and obtain the following equation: 
Table 1: Parameters of double pulsar PSR J0737-3039(Lyne et al. (2004); Burgay et al. (2003))

\begin{tabular}{clcccccccc}
\hline System & $M_{p}\left(M_{\odot}\right)$ & $M_{c}\left(M_{\odot}\right)$ & $P(\mathrm{~h})$ & $P_{s}(\mathrm{~ms})$ & $\mathrm{d}(\mathrm{kpc})$ & eccentricity & $\tau(\mathrm{yr})$ & $\dot{P}_{s}(\mathrm{~s} / \mathrm{s})$ & $B(\mathrm{G})$ \\
\hline $\mathrm{J} 0737-3039 \mathrm{~A}$ & $1.3381(7)$ & $\ldots \ldots \ldots \ldots$ & 2.448 & 22.699 & $1.15_{-0.15}^{+0.22}$ & 0.088 & $2.04 \times 10^{8}$ & $1.76 \times 10^{-18}$ & $6.4 \times 10^{9}$ \\
$\mathrm{~J} 0737-3039 \mathrm{~B}$ & $\ldots \ldots \ldots$. & $1.2489(7)$ & $\ldots \ldots \ldots$ & 2773.46 & $\ldots \ldots \ldots \ldots$ & $\ldots \ldots \ldots \ldots$ & $4.92 \times 10^{7}$ & $8.29 \times 10^{-16}$ & $1.59 \times 10^{12}$ \\
\hline
\end{tabular}

where $a_{0}$ is initial binary separation of DNS, and $T_{g w}$ is a characteristic time of GW induced coalescence, expressed as:

$$
T_{g w}=\frac{5}{32} \frac{M a_{0}^{4}}{\mu c R_{s}^{3}},
$$

with the Schwarzschild radius $R_{s}=2 G M / c^{2}$.

From Eq.(3) and Eq.(4), we can also deduce the relationship between orbital period and time as follows:

$$
P=P_{0}\left(1-\frac{t}{T_{g w}}\right)^{\frac{3}{8}},
$$

with $P_{0}$ the initial orbital period.

Now that we have the evolutionary equations of orbital scale and period induced by the GW radiation, the detailed simulations of double pulsars can be performed. PSR J0737-3039 is a DNS system where two pulsars were discovered by the pulsar search of Parkes 64 m radio telescope in 2003, lied in a distance of $1.15_{-0.15}^{+0.22} \mathrm{kpc}$ (Burgay et al. 2003; Lyne et al. 2004). The parameters of two NSs are noted below: PSR J0737-3039A is an old, fast-spinning, recycled pulsar, with mass of $1.3381 M_{\odot}$, the spin period of $22.7 \mathrm{~ms}$, and the spin period derivative is $1.76 \times 10^{-18} \mathrm{~s} / \mathrm{s}$ (Lyne et al. 2004); PSR J0737-3039B is a slow-spinning, non-recycled normal pulsar with a mass of $1.2489 M_{\odot}$, the spin period of $2773.46 \mathrm{~ms}$, and the spin period derivative is $8.29 \times 10^{-16} \mathrm{~s} / \mathrm{s}$ (Burgay et al. 2003). This system has also the shortest orbital period of 2.448 hrs among 19 pairs of DNSs, and the orbital eccentricity is as small as 0.088 , an almost circular orbit (Burgay et al. 2003). Moreover, the system presents a strong relativistic effect, orbit of which is confirmed to shrink by gravitational wave radiation during the evolution process (Kramer \& Wex 2009; van Leeuwen et al. 2015; Abbott et al. 2009).

Here, we simulate the orbital evolution of PSR J0737-3039 before the two NSs merge, where we assume that the two NSs are the point particles with the nearly circular orbit. Then, during the simulation, we regard that the DNS system begins to merge when the orbit radius decays to the scale of two NS radii $30 \mathrm{~km}$ with the conventional NS radius of $15 \mathrm{~km}$. Our simulation found that the merge age of PSR J0737-3039 system is $8.83 \times 10^{7} \mathrm{yr}$, and the maximum orbital frequency of the system before the merge is $590 \mathrm{~Hz}$, corresponding to the GW frequency as $1180 \mathrm{~Hz}$. A schematic diagram of orbital evolution of PSR J0737-3039 is plotted in Fig.1, where we draw a complete cycle of trajectory motion in every time interval $10^{7}$ yr. in-between $0-8 \times 10^{7} \mathrm{yr}$, and also include $8.8 \times 10^{7} \mathrm{yr}$ and $8.83 \times 10^{7} \mathrm{yr}$, and each trajectory gives the corresponding orbital period.

\section{THE EVOLUTION OF PSR J073-3039 SYSTEM IN COMPLETE COALESCENCE AGE}

Based on the magnetic dipole radiation model of pulsar (braking index $n=3$ ) (Lorimer et al. 2007; Shapiro \& Teukolsky 1983), we make a simple integral calculation for the pulsar spin-down model $\left(\dot{P}_{s} \propto P_{s}^{2-n}\right)$, 

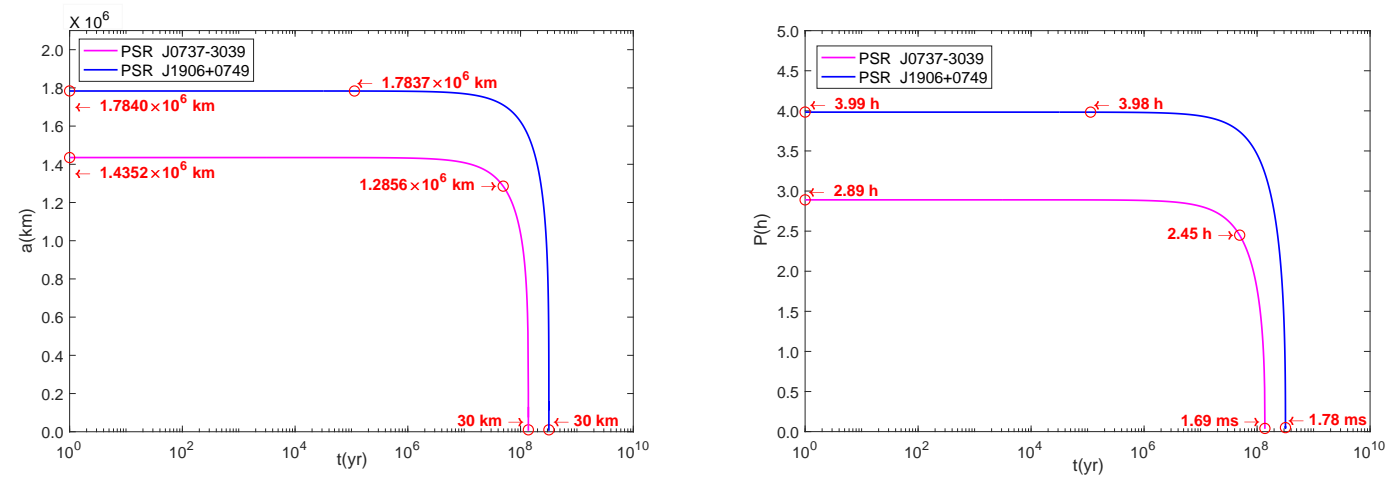

Fig. 2: The orbit evolution of double pulsar system PSR J0737-3039. As a comparison, PSR J0737-3039 and PSR J1906+0749 are indicated.

spin period $\left(P_{s 0}\right)$ of the pulsar, which can be written as (Lorimer et al. 2007; Shapiro \& Teukolsky 1983; Zhang et al. 2016):

$$
P_{s}^{2}=P_{s 0}^{2}+2 t\left[P_{s} \dot{P}_{s}\right]
$$

Since the normal pulsar (e.g., PSR J0737-3039B) has no experience of the accretion precess, its initial spin period is much smaller than its current spin period $\left(P_{s 0} \ll P_{s}\right)$, we can approximately deform Eq.(7) as:

$$
t \simeq \frac{P_{s}}{2 \dot{P}_{s}} \equiv \tau,
$$

where $\tau$ is the current characteristic age of the pulsar, expressed as $\tau \equiv P_{s} /\left(2 \dot{P}_{s}\right)$. The above formula indicates that we can use the characteristic age of pulsar to approximately replace its true age (when $P_{s 0} \ll P_{s}$ ) (Lorimer et al. 2007; Camilo et al. 1994). This means that the age of PSR J0737-3039 system is approximately equal to the current characteristic age of PSR J0737-3039B.

Compared to PSR J0737-3039A that experienced the accretion recycled process, PSR J0737-3039B is a relatively young normal pulsar with the spin period of $2773.46 \mathrm{~ms}$, with the characteristic age of $4.92 \times 10^{7}$ yr (Burgay et al. 2003). Similar to Crab pulsar that has the constant magnetic field dipole radiation (Staelin \& Reifenstein 1968), we assume that the initial spin period of PSR J0737-3039B is $20 \mathrm{~ms}$ (Zhang et al. 2016), because the current spin period is much larger than the birth spin period ( $20 \mathrm{~ms} \ll 2773.46 \mathrm{~ms}$ ), therefore, consequently the PSR J0737-3039B true age $\left(t_{B}\right)$ can be approximated by the characteristic age $\left(\tau_{B}\right)$, namely:

$$
t_{B} \simeq \tau_{B} .
$$

Lorimer et al. (2007) pointed out that when the PSR J0737-3039A ceased to spin-up and the PSR J07373039B was birth occurred almost at the same time (Lorimer et al. 2007), thus the spin-down age of PSR J0737-3039A (time since the accretion induced spin-up stopped: $t_{A}$ ) can be approximately replaced by the true age of PSR J0737-3039B, that is:

$$
t_{A}=t_{B} \simeq \tau_{B}
$$

According to the standard DNS formation model (van den Heuvel 2007), the first NS of the system was formed by a massive star undergoing main sequence evolution and supernova explosion. The first NS will 
NS; If the system survives in the second supernova explosion, the DNS system will be born at this moment. Hence the true age of the DNS system is equal to the true age of the second NS, and the true age of PSR J0737-3039 system $\left(t_{A B}\right)$ :

$$
t_{A B}=t_{B} \simeq \tau_{B}
$$

from the above formula we can constrain the real age of PSR J0737-3039 system to be $4.92 \times 10^{7} \mathrm{yr}$, and the system will complete coalescence age (time from birth to merger) in approximately $1.38 \times 10^{8} \mathrm{yr}$ (the characteristic age of PSR J0737-3039B to be $4.92 \times 10^{7} \mathrm{yr}$, the coalescence age of system to be $1.38 \times 10^{8}$ yr).

We bring the age of PSR J0737-3039 system $t_{A B}$ into Eq.(4) and Eq.(6), and obtain the initial binary separation of the system to be $1.44 \times 10^{6} \mathrm{~km}$, corresponding to be initial orbital period of $2.89 \mathrm{hrs}$, which means that the binary separation and orbital period of system is only contracted by $0.15 \times 10^{6} \mathrm{~km}$ and $0.44 \mathrm{hrs}$, respectively (the present binary separation and orbital period are $1.29 \times 10^{6} \mathrm{~km}$ and $2.45 \mathrm{hrs}$, respectively). Next, based on the initial information of PSR J0737-3039 system, we simulated the evolution of the orbital period and binary separation of PSR J0737-3039 within the complete coalescence age, as shown in Fig.2.

\subsection{The spin evolution of PSR J0737-3039A and PSR J0737-3039B in complete coalescence age}
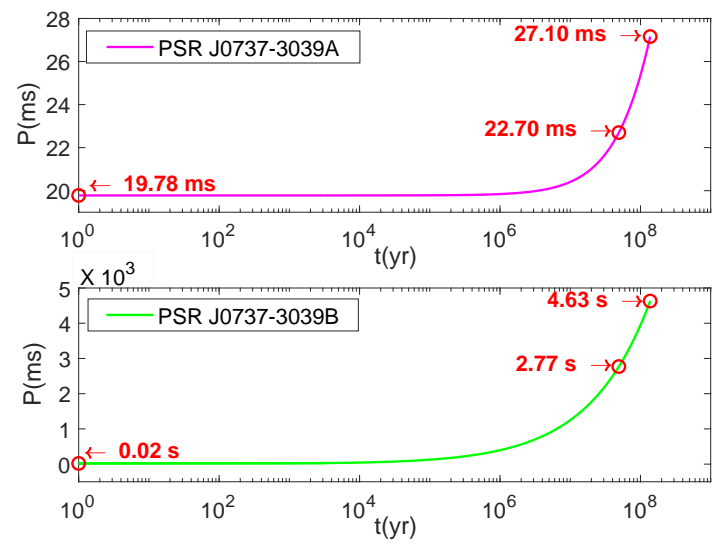

Fig. 3: Spin period evolutions of PSR J0737-3039A and PSR J0737-3039B.

We bring the observational data of PSR J0737-3039A and $t_{A}$ into Eq.(7), obtaining that the birth spin period is $19.78 \mathrm{~ms}$ when it starts to spin-down, which means that its spin period is only increased by 2.92 ms (the present spin period is $22.70 \mathrm{~ms}$ ). From the above, the initial spin period of PSR J0737-3039B is assumed to be similar to that of the Crab-pulsar of $20 \mathrm{~ms}$, compared to its current period of $2773.46 \mathrm{~ms}$, thus the spin period of PSR J0737-3039B evolves relatively quickly.

In order to simulate the evolution of the pulsar spin-down, we assume that the dipole magnetic field is invariant during the evolution, therefore we can transform Eq.(7) into:

$$
P_{s}^{2}=P_{0}^{2}+2 t\left[P_{n} \dot{P}_{n}\right],
$$

where $P_{n}$ and $\dot{P}_{n}$ are the current measured data of the pulsar spin period and spin period derivative, respec- 
According to Eq.(12), we simulated the spin period evolution of PSR J0737-3039A and PSR J07373039B within the complete merger age, and stopped the simulation when the evolution time going to $1.38 \times$ $10^{8} \mathrm{yr}$. Our simulation found that: the spin period of PSR J0737-3039A and PSR J0737-3039B are 27.10 $\mathrm{ms}$ and $4.63 \mathrm{~s}$, respectively, when the system was merged. Based on the simulation results, we plot the spin period evolution of PSR J0737-3039A and PSR J0737-3039B in Fig.3.

In order to acquire a complete scenario of the evolution of both pulsars in the diagram of spin period and magnetic field (the magnetic fields of PSR J0737-3039A and PSR J0737-3039B are $6.4 \times 10^{9} \mathrm{G}$ and $1.59 \times 10^{12} \mathrm{G}$, respectively (Burgay et al. 2003; Lyne et al. 2004), we plot their complete simulation results in Fig.4.

In the Figure 4, the small box is a close-up of the evolution track of PSR J0737-3039A, and the arrows indicate the direction of evolution (the evolution from left to right means that from birth to merger). It can be seen from the figure that from the system birth to merger, PSR J0737-3039A would be always seen by the radio telescope, while PSR J0737-3039B will cross the death line of pulsar and may be not observed (Bhattacharya \& van den Heuvel 1991).

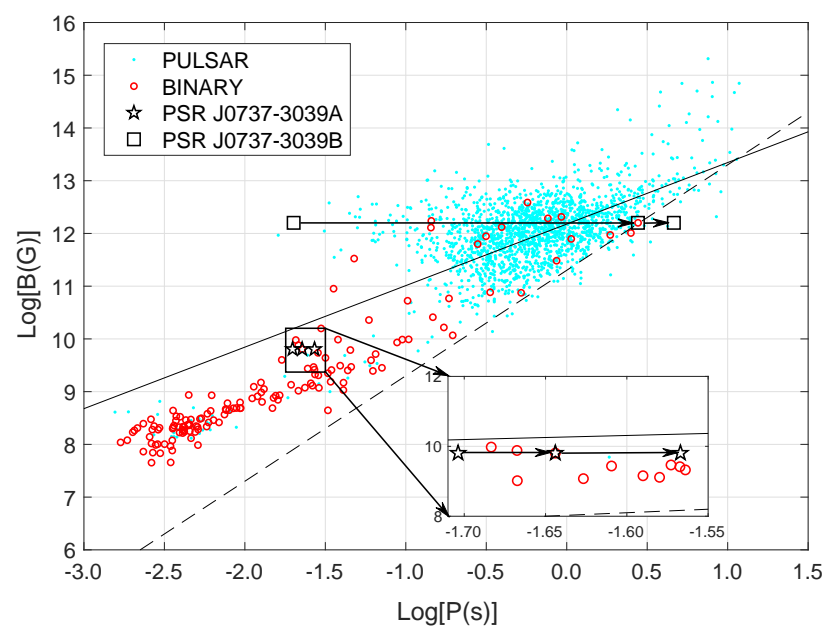

Fig. 4: The evolution track of PSR J0737-3039A and PSR J0737-3039B along the $B-P$ diagram (data from ATNF pulsar catalog (Manchester et al. 2005). The cyan dots, red circles, black lines, black dotted lines, pentagrams and squares are represent normal pulsars, pulsed binary, acceleration lines, death lines, PSR J0737-3039A and PSR J0737-3039B, respectively.

\section{THE RESULTS AND DISCUSSIONS}

In this paper, based on the observational data of the double pulsar system PSR J0737-3039, we simulated its orbital evolution induced by the GW radiation, and found that the system will merge after $8.83 \times 10^{7} \mathrm{yr}$. By the magnetic dipole model of pulsar, we obtained that PSR J0737-3039 system exists about $4.92 \times 10^{7}$ yr, thus the complete coalescence age (from its birth to merge) of PSR J0737-3039 system should be be $1.38 \times 10^{8} \mathrm{yr}$. Next, we simulated the complete orbital evolution of PSR J0737-3039 system and obtained the initial orbital period and radius as $2.89 \mathrm{hrs}$ and $1.44 \mathrm{~km}$, respectively. In addition, the GW frequency 
binary separation decays from $442 \mathrm{~km}$ to $30 \mathrm{~km}$. We compared the GW frequency evolution of PSR J107373039 with that of the observation data of GW170817 by LIGO, as shown in Fig.5, and found that both curves have no much difference, and a meager bias is on account of the mass difference of both systems (the primary NS and companion NS masses of GW170817 are: $1.46_{-0.10}^{+0.12} M_{\odot}$ and $1.27_{-0.09}^{+0.09}$, respectively (Abbott et al. 2018).

Furthermore, we calculated the spin period evolution of PSR J0737-3039A and PSR J0737-3039B within the complete merger age, and obtained that their spin periods are $27.10 \mathrm{~ms}$ and $4.63 \mathrm{~s}$ at coalescence. Through the evolution trajectories of PSR J0737-3039A and PSR J0737-3039B in the pulsar magnetic field and spin period $B-P$ diagram, we concluded that PSR J0737-3039A can always be observed by radio telescopes, but PSR J0737-3039B will cross the death line of radio pulsar and could not be observed in general.

It is remarked that we employed the point masses of both NSs to perform the simulation. However, Kuznetsov et al. (1998) studied the orbital evolution of DNS by the GW radiation while the internal structure of NSs have been taken into account, and pointed out that there exists little effect on their merging time (only deviation of $10.5 \mathrm{~ms}$ ) (Kuznetsov et al. 1998), which is much less than the merging time of $30 \mathrm{~s}$ as observed in GW170817 by LIGO.

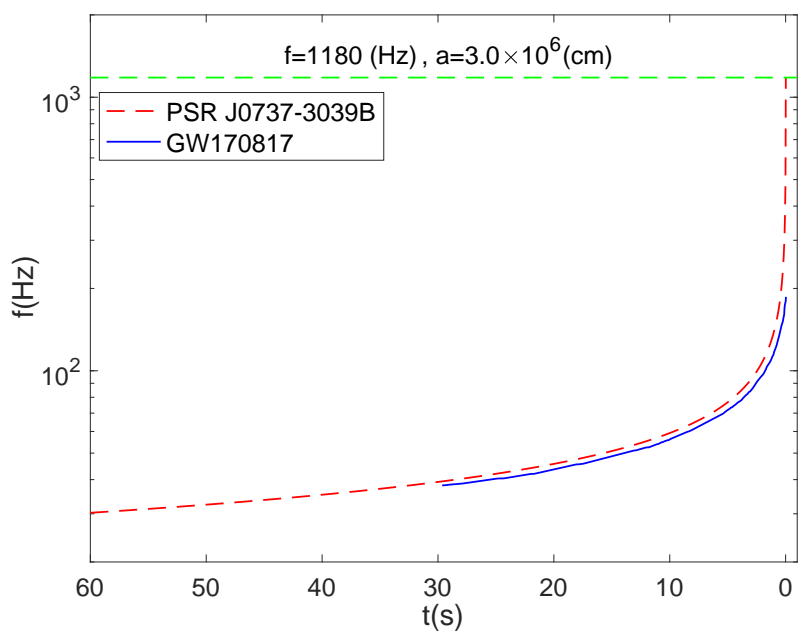

Fig. 5: The evolution of GW frequency at the last minute of DNS merge, where the dashed (solid) curve represents the case of PSR J0737-3039 (GW170817).

Acknowledgements This research is supported by the National Natural Science Foundation of China NSFC (11988101, 11773005, U1631236, 11703001, U1731238, U1938117, 11725313, 11721303), the International Partnership Program of Chinese Academy of Sciences grant No. 114A11KYSB20160008, the National Science Foundation of China No. 11721303, and the National Key R\&D Program of China No. 2016YFA0400702. And, this work is also supported by the National Basic Research Program (973 Program) (no. 2015CB857100), National Key R\&D Program of China (no. 2017YFA0402600), and the 


\section{References}

Abbott B. P., Abbott R., Abbott T., D. et al. 2017, Phys. Rev. Lett, 119, 1611012

Abbott B. P., Abbott R., Adhikari R., et al. 2009, Phys. Rev. D, 79, 1220014

Abbott B. P., Abbott R., Abbott T. D., et al. 2018, Phys. Rev. X, 9, 0310408

Blanchard P. K., Berger E., Fong W., et al. 2017, Astrophys. J, 848, L22 2

Burgay M., D'Amic N., Possenti A., et al. 2003, Nature, 426, 531 2, 4, 5,7

Bhattacharya D., \& van den Heuvel E. P. J. 1991, Phys. Rev, 203, 1 2, 7

Cutler C., Apostolatos T. A., Bildsten L., et al. 1992, Astrophys. J, 70, 29842

Camilo F., Thorsett S. E., \& Kulkarni S. R. 1994, Astrophys. J, 421, L15 2, 5

Einstein A., \& Sitzungsber K. 1916, Preuss. Akad. Wiss, 1, 6881

Hulse R. A., \& Taylor J. H. 1975, Astrophys. J, 195, L51 1

Kramer M., Sarkissian J., Freire P. C. C., et al. 2004, eConf C041213, 00382

Kramer M., Stairs I. H., Manchester R. N., et al. 2006, Science, 314972

Kramer M., \& Wex N. 2009, CQGra, 26, 073001 2, 4

Kuznetsov O. A., Prokhorov M. E., Sazhin M. V., \& Chechetkin V. M. 1998, Astron. Rep, 42, 6388

Lyne A. G., \& Graham-Smith F. 2012, Pulsar Astronomy (Cambridge University Press, 2012) 2, 3

Lightman A. P., Press W. H., Price R. H., et al. Problem book in relativity and gravitation (Princeton University Press, 1975) 3

Lightman A. P., \& Shapiro S. L. 1975, Astrophys. J, 198, L73 3

Lorimer D. R., Freire P. C. C., StairsI. H., et al. 2007, Mon. Not. R. Astron. Soc, 379, 1217 2, 4, 5

Lyne A. G., Burgay M., Kramer M., et al. 2004, Science, 303, 1153 2, 4, 7

Maione F., Pietri R. D., Feo A., \& Löffler F. 2016, CQGra, 33, 1750092

Manchester R. N., Hobbs G. B., Teoh A., et al. 2005, Astrophys. J, 129, 19937

Nomoto K. 1984, ApJ, 277, 7912

Ohanian H. C., \& Ruffini R. Gravitation and Spacetime (W. W. Norton \& Company, 1994). 3

Podsiadlowski P., Langer N., Poelarends A. J. T., et al. 2004, ApJ, 612, 10442

Peters P. C., \& Mathews J. 1963, Phys. Rev, 131, 4353

Peters P. C. Phys. Rev. Lett, 1964 136, 1224 2, 3

Staelin D. H., \& Reifenstein E. C. 1968, Science, 162, 14815

Schutz B. F. 1986, Nature, 323, 3102

Shibata M., \& Uryu K. 2000, Phys. Rev. D, 61, 0640012

Shapiro S. L., \& Teukolsky S. A. 1983, Black Holes, White Dwarfs, and Neutron Stars: The Physics of Compact Objects. John (Wiley, 1983) 3, 4, 5

Taylor J. H., \& Weisberg J. M. 1982, Astrophys. J, 253, 9082

Troja E., Piro L., van Eerten H., et al. 2017, Nature, 551, 712

Tauris T. M., Langer N., Moriya T. J., et al. 2013, ApJ, 778, L23 2

Tauris T. M., Langer N., \& Podsiadlowski P. 2015, MNRAS, 451, 21232

Tauris T. M., Kramer M., Freire P. C. C., et al. 2017, ApJ, 846, 1702

van den Heuvel E. P. J. ArXiv Astrophysics e-prints. (2007) [arXiv:0704.1215 [astro-ph]]. 5 
van den Heuvel E. P. J. 2004, Science, 303, 11432

van Leeuwen J., Kasian L., Stairs I. H., et al. 2015, Astrophys. J, 798, 1184

Yang Y. Y., Zhang C. M., Li D., et al. 2017, Astrophys. J, 835, 1852

Zhang C. M., Zhang Y. Z., Gong Y. Q., et al. 2016, ASPC, 520, 255 